\title{
Flexible bi-layer terahertz chiral metamaterials
}

\author{
Amornthep Sonsilphong ${ }^{1}$, Philipp Gutruf ${ }^{2}$, \\ Withawat Withayachumnankul ${ }^{2,3}$, Derek Abbott ${ }^{3}$, Madhu Bhaskaran ${ }^{2}$, \\ Sharath Sriram ${ }^{2}$ and Nantakan Wongkasem ${ }^{1}$
}

\author{
${ }^{1}$ Metasolver Laboratory and Department of Electrical Engineering, Faculty of Engineering, Khon Kaen \\ University, Khon Kaen, 40002, Thailand \\ ${ }^{2}$ Functional Materials and Microsystems Research Group, RMIT University, Melbourne, Victoria, 3001, \\ Australia \\ ${ }^{3}$ School of Electrical and Electronic Engineering, The University of Adelaide, Adelaide, SA 5005, \\ Australia \\ E-mail: nantakan@kku.ac.th
}

Received 23 March 2015, revised 2 June 2015

Accepted for publication 3 June 2015

Published 20 July 2015

\begin{abstract}
Thin and flexible bi-layer terahertz chiral metamaterials are experimentally demonstrated in both handednesses at terahertz frequencies. The structures are made of conjugated $\mathrm{C} 8$ metallic resonators fabricated on a flexible polyimide substrate. These structures exhibit chirality and can distinguish circularly polarized waves at the frequency range from 0.5 to $0.9 \mathrm{THz}$, equivalent to $57.15 \%$ bandwidth. The structures can be used as circular polarizers for terahertz waves.
\end{abstract}

Keywords: metamaterials, chiral media, flexible, negative refractive index

\section{Introduction}

Optical rotary dispersion (ORD) or optical activity, and circular dichroism (CD) [1] are the two unique electromagnetic (EM) features of chiral media. As an arbitrary EM wave enters chiral media, the wave will be decomposed into two eigenmodes, right and left circularly polarized waves (RCP and LCP waves). Because of ORD, the two waves propagate inside the media with different velocities ORD. This difference causes the rotation of the plane of polarization. Additionally, due to $\mathrm{CD}$, the RCP and LCP waves will later be combined into an elliptically polarized wave at the outgoing surface [2]. The sensitivity of rotation to the polarization state can have a significant impact on several optical applications, such as broadband circular polarizers [3], switchable and tunable circular polarizers [4] and sensors [5].

Chirality can be naturally found in solid materials and organic molecules such as quartz and DNA at different spectral ranges depending on their crystalline or molecular unit cell size [2]. However, chirality is normally scarcely exhibited at terahertz and optical frequencies. Therefore, recently, artificial chiral structures or chiral metamaterials (CMMs) have been proposed and demonstrated to achieve strong chirality at any desired frequency. CMMs are composed of arrays of conductive resonators that cannot be superimposed onto their mirror images [1]. Several CMMs have been studied in the microwave regime, such as Swiss rolls [6, 7], planar helices [8, 9], chiral SRRs [10], and multilayer rosettes structures [11]. In particular, conjugated bi-layer C8 structures have been investigated in the microwave regime $[12,13]$. It has been found that chirality and refractive index of these $\mathrm{C} 8$ structures can be controlled effectively via the structures' geometry. Extreme chirality results in a strong negative refractive index for both RCP and LCP excitations [13]. At terahertz frequencies, strong optical activity and negative refractive index were exhibited in the free-standing meta-foils consisting of a self-supported square array of interconnected conjugated rosettes [14]. Threedimensional helix structures [3] were fabricated using direct laser writing and electrochemical deposition with a limited sample size due to the serial writing process. Planar CMMs [15, 16], I-shape [17], gammadion [18], Y-shape [19], twisted-arc [20], and several flexible chiral structures [21] fabricated using photolithography, electron-beam lithography, and electron beam evaporation were also introduced at terahertz frequencies.

In this paper, we focus on controlling the polarization properties of the conjugated bi-layer right-handed $(\mathrm{RH})$ and 


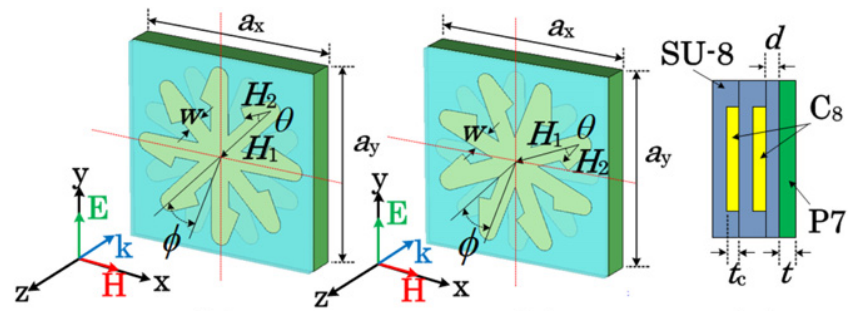

(a)

(b)

(c)

Figure 1. Unit cells of conjugated bi-layer structures. (a) RH, (b) LH C8 structures, and (c) the side view with the dimensions:

$H_{1}=75 \mu \mathrm{m}, H_{2}=30.04 \mu \mathrm{m}, a_{x}=a_{y}=185 \mu \mathrm{m}, w=18 \mu \mathrm{m}, t=25 \mu \mathrm{m}$, $t_{c}=0.2 \mu \mathrm{m}$, and $d=2.5 \mu \mathrm{m} . \phi=22.5^{\circ}$ and $\theta=22.5^{\circ}$.

left-handed (LH) C8 structures in the terahertz frequency regime. The transmission coefficients, refractive indices and chirality of the structure in response to RCP and LCP waves are investigated. The structures are designed with particularly suitable materials in order to establish chirality with low loss in the terahertz regime. In order to enable the designed structures to be used in a variety of applications, thin and flexible substrates are selected, and the unconventional thin-film fabrication process is implemented [22]. The flexibility might add the tunability for these proposed CMMs [23]. Coherent broadband terahertz time-domain spectroscopy (THz-TDS) is used for characterization of the fabricated chiral samples.

\section{Flexible bi-layer CMMs}

The proposed unit cells for the two handedness structures are illustrated in figure 1 , along with the propagation direction, $\vec{k}$, electric field, $\vec{E}$, and magnetic field, $\vec{H}$. Two conjugated C8 structures are located on top of each other, with a twist angle $\varphi$. The arms of each structure lay in the opposite direction as 'the mirror' twisted layout. To generate the RH and LH handedness, the arms of the front structure are set in the counter clockwise and clockwise directions, respectively. $H_{1}$ and $H_{2}$ indicate the main axis and the arm of the structures, respectively. The angle between $H_{1}$ and $H_{2}$ is $\theta$. The side view of the unit cell $\mathrm{C} 8$ is shown in figure 1(c). A flexible polyimide (PI) film, and photoresist SU-8 layers are respectively used as the substrate and the spacers for the $\mathrm{C} 8$ structure. The complex permittivities of PI and SU-8 are, respectively $\varepsilon_{\mathrm{PI}}=3.03+0.36 \mathrm{i}$ and $\varepsilon_{\mathrm{SU}-8}=2.90+0.41 \mathrm{i}$ at $1.0 \mathrm{THz}[24,25]$. As a complementary to the experimental validation, simulation results for the transmission and reflection properties and field distributions are obtained from CST Microwave Studio. In the simulations, the bi-layer unit-cell structures are modeled by using a periodic boundary condition to mimic an infinite $2 \mathrm{D}$ array. The surface impedance model is applied to the metal to account for its loss at terahertz frequencies.

Based on the synthesis method presented in [22], a technique to attain a multilayer design on polyimide, suitable for terahertz applications is developed. The production process starts with the preparation of the rigid carrier, a silicon wafer. A layer of $2.5 \mu \mathrm{m}$ is applied by spinning the SU-8 2002 photoresist. The negative photoresist is prebaked and exposed to UV light, successively the substrate; a commercial polyimide film (PC500FILM-100-108) with a thickness of $25.4 \mu \mathrm{m}$ is laminated to the SU-8 coated silicon wafer. The lamination process is carried out using a standard office laminator with an elevated temperature of $100{ }^{\circ} \mathrm{C}$, illustrated in figure 2(a). At this temperature the SU-8 becomes slightly viscous and allows the polyimide film to be temporarily attached to the silicon wafer. After cooling down, the SU-8 is also fully cross-linked by passing through the laminator, eliminating the need for development. The cross-linking is essential to endure the following production steps. This novel lamination process enables the polyimide substrate to attain full contact with the mask during hard contact photolithography to achieve high resolution features. Note that no curing of the polyimide layer needed as the production process is for an off-the-shelf polyimide film. This allows for rapid fabrication due to the elimination of the elaborate curing step of polyimide films. Subsequently a $2.5 \mu \mathrm{m}$ thick layer of SU-8 is spun on the polyimide followed by standard microfabrication methods. There is no remaining SU-8 on the backside of the sample due to a much higher adhesion of the SU-8 film to the silicon wafer than to the polyimide film. To pattern the gold features a standard lift off process was carried out using an AZ 5214E photoresist in image reversal mode with a following e-beam evaporation at a rate of $1 \AA \mathrm{s}^{-1}$ of $20 \mathrm{~nm}$ chromium as an adhesion layer on either side of the $200 \mathrm{~nm}$ gold layer, followed by an acetone lift off, depicted in figures 2(b) and (g). After that, to complete the structures, the previous processes are repeated successively, illustrated in figures 2(c)-(e) for LH media, and figures 2(h)-(j) for RH media. The fabrication is completed with the release of the sample from its SU-8 coated silicon carrier by carefully lifting the polyimide layer with a razorblade which allows an easy release once the adhesion is firstly overcome, shown in figures 2(f) and (k) for $\mathrm{LH}$ and $\mathrm{RH}$ media respectively. The resulting device is displayed in figure 3 , a high resolution optical microscope image. It can be clearly seen that the photolithographic step is not compromised by the flexible substrate, both alignment and resolution are comparable to processing on silicon substrate.

The measurement is then carried out on the fabricated samples by using coherent broadband THz-TDS. The fibercoupled Tera K15 by Menlo Systems GmbH is employed with a bandwidth spanning about $0.2-1.5 \mathrm{THz}$. Four identical lenses couple terahertz radiation between the emitter and receiver to form a focused beam with a diameter of a few millimeters. For each CMMs sample, four transmission scans are taken at normal incidence in both the co-and cross-polarizations. The polarimetric analysis is carried out via two polarizers, affixed to the polarization-sensitive emitter and detector, to improve the polarization purity. The results are then normalized with a freespace co-polarized transmission scan to deliver complex transmission responses, $T_{x x}, T_{x y}, T_{y x}$, and $T_{y y}$. 


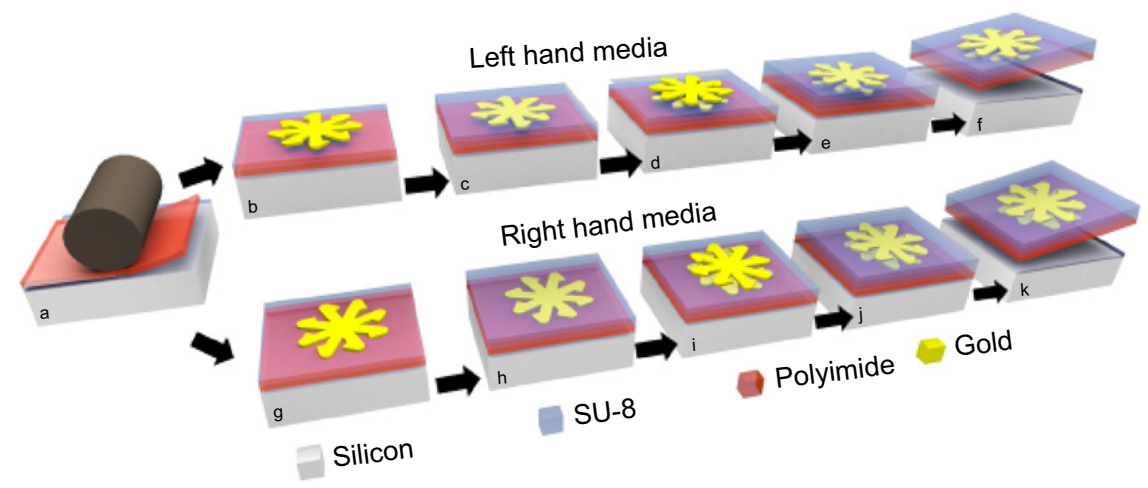

Figure 2. Schematic of the fabrication process.
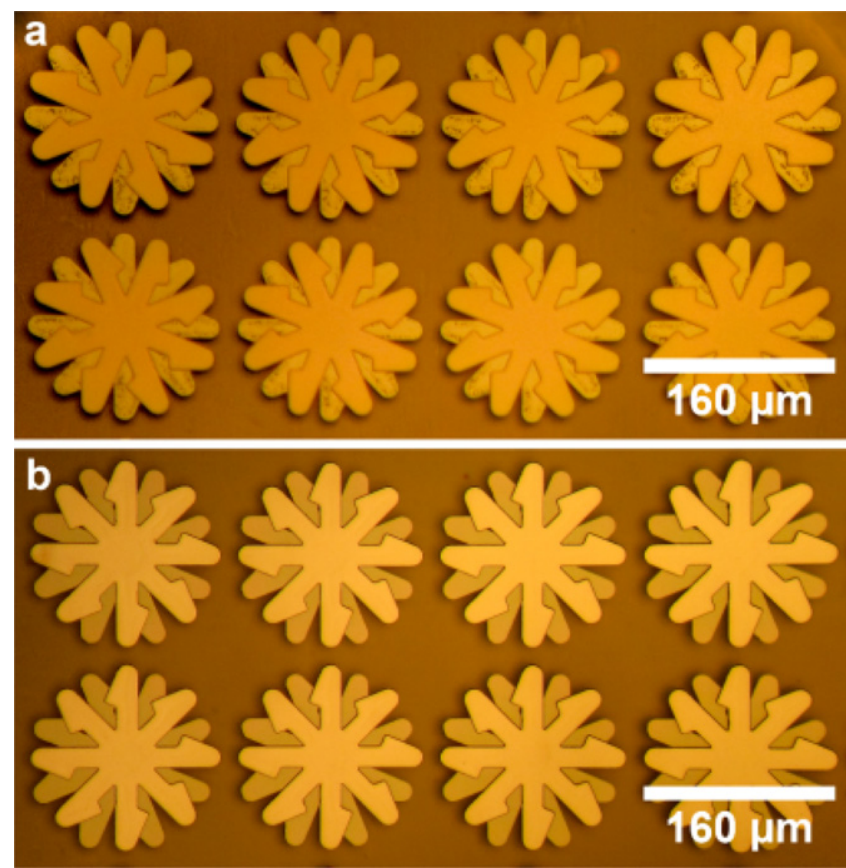

Figure 3. Optical micrographs of the fabricated left-handed metamaterial (a) and right-handed metamaterial (b).

These measured linear co-and cross-polarization transmission responses of the conjugated bi-layer $\mathrm{C} 8$ structure, $\mathrm{RH}$ and $\mathrm{LH}$, are comparable to those from the simulation. As shown in figures 4(a) and (b), the measured co-polarized responses $\left(T_{x x}\right.$ and $\left.T_{y y}\right)$ of both the RH and LH C8 structures show the resonance at $0.7 \mathrm{THz}$. Additionally, the magnitude of the linearly cross-polarized responses $\left(T_{x y}\right.$ and $\left.T_{y x}\right)$ shown in figures 4(c) and (d) of both handedness is around 0.05, spanning $0.6-1.0 \mathrm{THz}$. It could be seen that the linear-polarization responses of the RH and LH media are almost identical. The resonance location of these responses indicates the location of chirality. Here, the dissipation loss of the substrate and the spacers plays a significant role in these weak crosspolarization responses, and will be further investigated through simulations.

The complex transmission coefficients of RCP and LCP waves, $T_{++}$and $T_{--}$, can be obtained from the linear

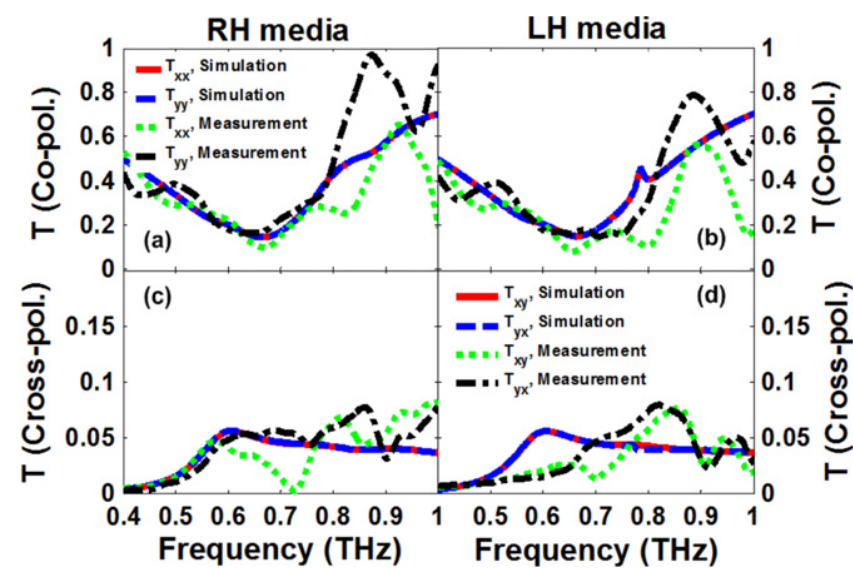

Figure 4. Magnitude of linearly co- and cross-polarized responses of (a) and (c) conjugated bi-layer RH structure, and (b) and (d) conjugated bi-layer LH C8 structure.

polarization responses [26]:

$$
\left(\begin{array}{c}
T_{++} \\
T_{--}
\end{array}\right)=\frac{1}{2}\left(\begin{array}{l}
\left(T_{x x}+T_{y y}\right)+\mathrm{i}\left(T_{x y}-T_{y x}\right) \\
\left(T_{x x}+T_{y y}\right)-\mathrm{i}\left(T_{x y}-T_{y x}\right)
\end{array}\right) .
$$

Figure 5 shows a comparison between simulation and measurement results of the conjugated bi-layer $\mathrm{RH}$ C8 structure. The transmission coefficients of the RCP and LCP waves are presented in figures $5(\mathrm{a})$ and (b). The transmission for the RCP wave is higher than that for the LCP wave at the observed resonance for both the simulation and measurement results. This confirms the RCP transmission in the RH chiral structure. The opposite behavior is found in the LH structure, as confirmed by the results shown in figures 6(a) and (b). Additionally, the polarization changes in a linearly polarized wave exciting the $\mathrm{RH}$ and $\mathrm{LH}$ C8 chiral structures are investigated. Figures 5(c)-(f) and 6(c)-(f) show the polarization changes in terms of the ellipticity $\eta$ and the polarization azimuth rotation angle $\theta$ of the transmitted wave. Both $\eta$ and $\theta$ from the simulation and measurement results agree well for both the RH and LH structures. The effects of anisotropic phase shift and absorption can be also retrieved using the generalized matrix equivalence theorem [27]. 


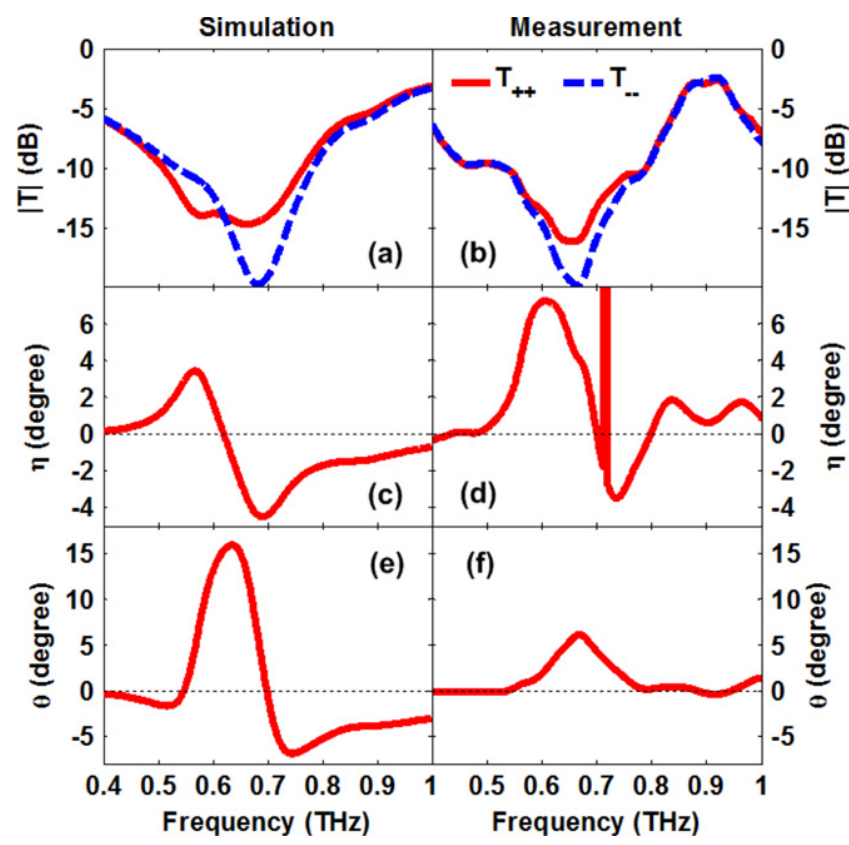

Figure 5. Simulation (left) and measurement (right) results of the conjugated bi-layer RH C8 structure. (a) and (b) The transmissions of the RCP and LCP waves. (c) and (d) The ellipticity $\eta$ of the transmitted wave. (e) and (f) The polarization azimuth rotation angle $\theta$.

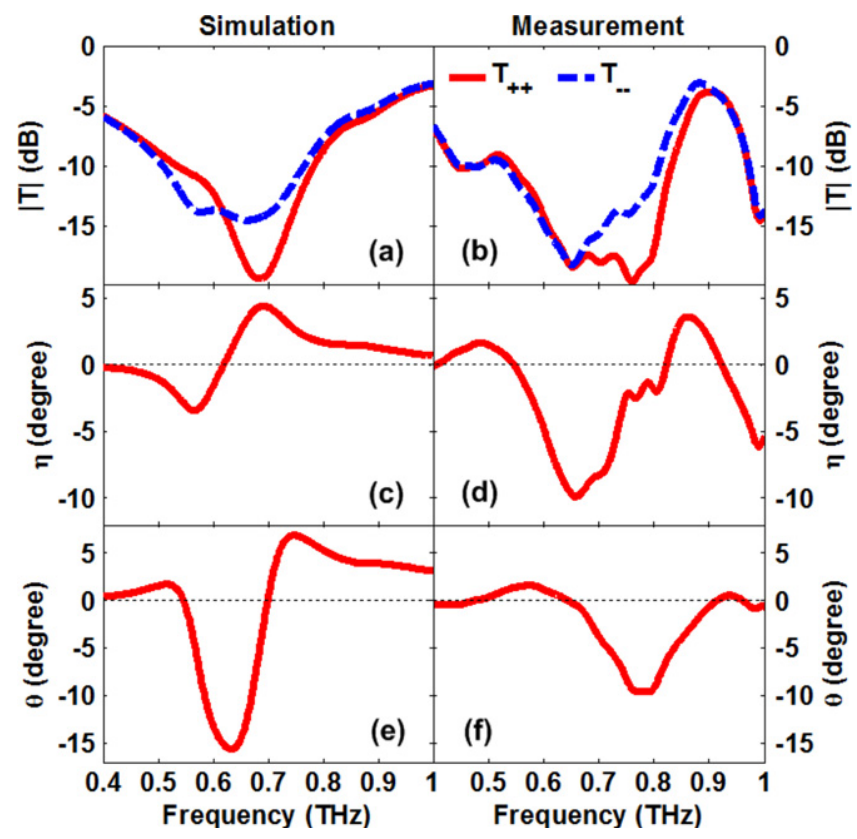

Figure 6. Simulation (left) and measurement (right) results of the conjugated bi-layer LH C8 structure. (a) and (b) The transmissions of the RCP and LCP waves. (c) and (d) The ellipticity $\eta$ of the transmitted wave. (e) and (f) The polarization azimuth rotation angle $\theta$.

The simulation results of the conjugated bi-layer $\mathrm{RH}$ and LH C8 structures with lossy dielectric are presented in figure 7. Within the frequency of interest, the transmission amplitude responses for the RCP and LCP excitations are

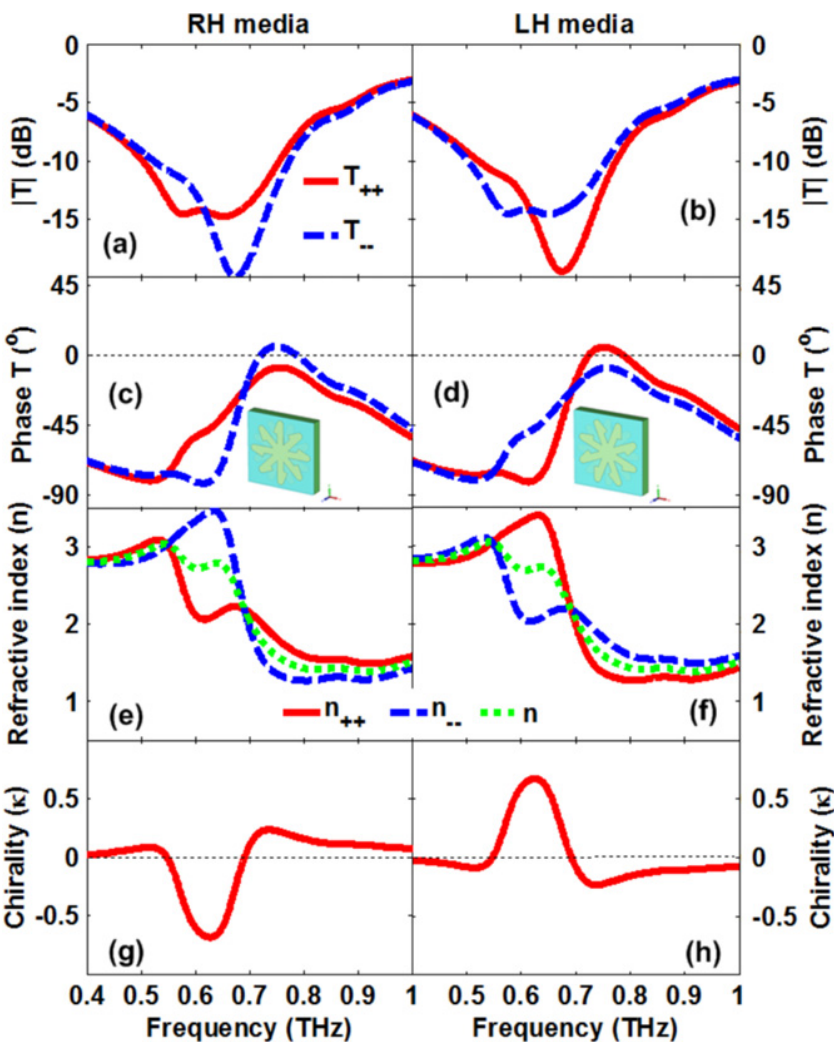

Figure 7. Properties of the conjugated bi-layer RH and LH C8 structures with lossy dielectrics in response to the circularly polarized waves. (a)-(b) Transmission amplitude and (c)-(d) transmission phase, (e)-(f) refractive index, and $(\mathrm{g})-(\mathrm{h})$ chirality.

different. For the RH structure, a resonance dip for the LCP wave can be found at $0.68 \mathrm{THz}$, as shown in figure 7(a). In contrast, the transmission coefficient of the RCP wave in the same frequency range is slightly higher. It is clear that the structures can discriminate the two circularly polarized waves, since the $\mathrm{CD}$ can be observed. The frequency band of this different response from the two $\mathrm{CP}$ waves is significantly broad, from 0.5 to $0.9 \mathrm{THz}$ or $57.15 \%$ bandwidth. Due to the phase difference (figure 7(c)) between the responses of the $\mathrm{RCP}$ and LCP waves, the refractive indices, $n_{++,--}=n \pm \kappa$, of the RCP and LCP waves (figure 7(e)) are degenerated. $n_{++,--}$can be obtained from the transmission and reflection coefficients [25]. The degeneration of the refractive indices confirms the chirality, $\kappa=\left(n_{++}-n_{--}\right) / 2$, as shown in figure $7(\mathrm{~g})$. The conditions for the RCP and LCP waves are reversed in the case of the LH structure, as can be seen from the results shown in figures 7(b), (d), (f) and (h).

Figure 8 presents the current distributions of the LH conjugated bi-layer C8 structures at 0.45 and $0.58 \mathrm{THz}$ under the RCP and LCP excitations. The current direction on the top layer is the same as the one on the bottom layer indicating a parallel current mode, illustrated in figures $8(\mathrm{a})$ and (b) at $0.45 \mathrm{THz}$. In figures $8(\mathrm{c})$ and (d), there is an antipallarel current at $0.58 \mathrm{THz}$ since the current direction on the top and bottom layer is opposite. This also denotes an asymmetric resonance mode or coupled magnetic dipole resonance [28- 


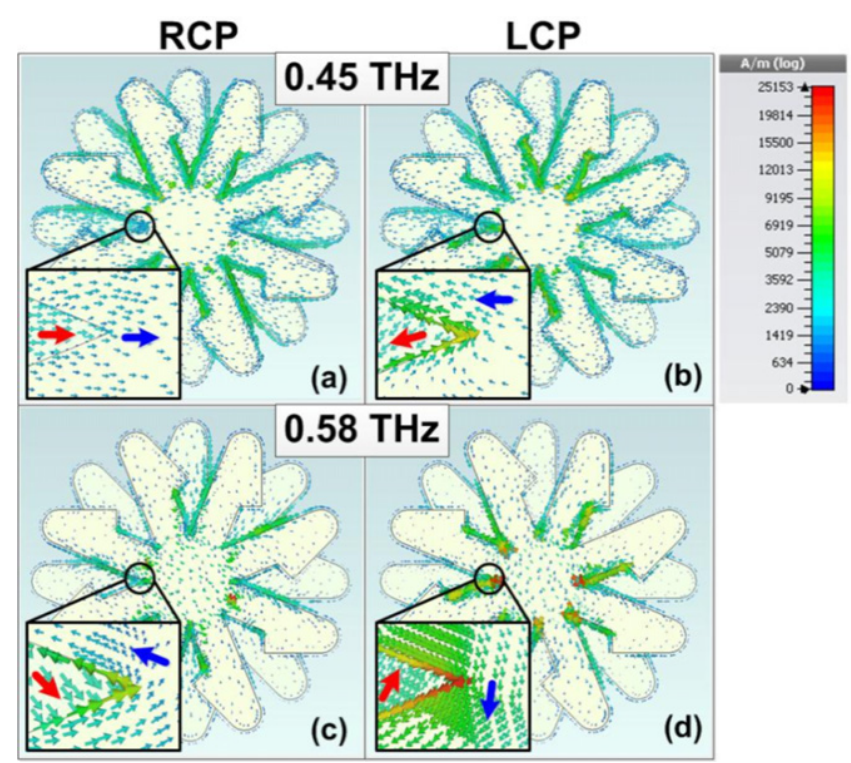

Figure 8. Current distributions of $\mathrm{LH} \mathrm{C} 8$ structures at $0.45 \mathrm{THz}$ from (a) RCP and (b) LCP excitation, and at $0.58 \mathrm{THz}$ from (c) RCP and (d) LCP excitation.

30]. These results support the chirality appearance of these LH C8 structures as shown in figure 7(h).

It is important to stress that if dielectric losses of materials are not taken into consideration, the responses for the circularly polarized waves would be considerably different. Figure 9 shows the calculated complex transmission profiles, refractive indices, and chirality of $\mathrm{C} 8$ structures with lossless dielectrics. There is a strong chirality band from 0.53 to $0.80 \mathrm{THz}$. For the RH C8 structures, within the considered polarization conversion band, the transmission response of the RCP wave is improved. Two transmission peaks of the RCP wave are formed at 0.59 and $0.70 \mathrm{THz}$. Note that due to the large chirality at the second peak at $0.7 \mathrm{THz}$, a negative refractive index is observed. Also loss factors, $\mathrm{LF}=\operatorname{Im}(n) / \mathrm{Re}$ (n), are studied and shown in figures 9(i) and (j); Note that LF indicates transmission properties. When LF is close to zero, it specifies low loss. It can be seen that LF of the LCP wave is less than 0.2 at $0.59 \mathrm{THz}$.

To improve the polarization conversion efficiency, the permittivity and thickness of the substrate and spacers are taken into consideration. Figure 10 illustrates the transmission and material properties of the conjugated bi-layer LH C8 structures. The permittivity of the spacers is now equal to 1.5 , and that of the substrate is slightly higher at 3.0. The thickness of the substrate is $20 \mu \mathrm{m}$, compared to $25.4 \mu \mathrm{m}$ for the fabricated structure. The first transmission peak of the RCP wave at $0.57 \mathrm{THz}$ is now $-6.94 \mathrm{~dB}$, improving by $55 \%$ as compared to our original design shown in figure 9 . The negative refractive index can also be observed at the second peak.

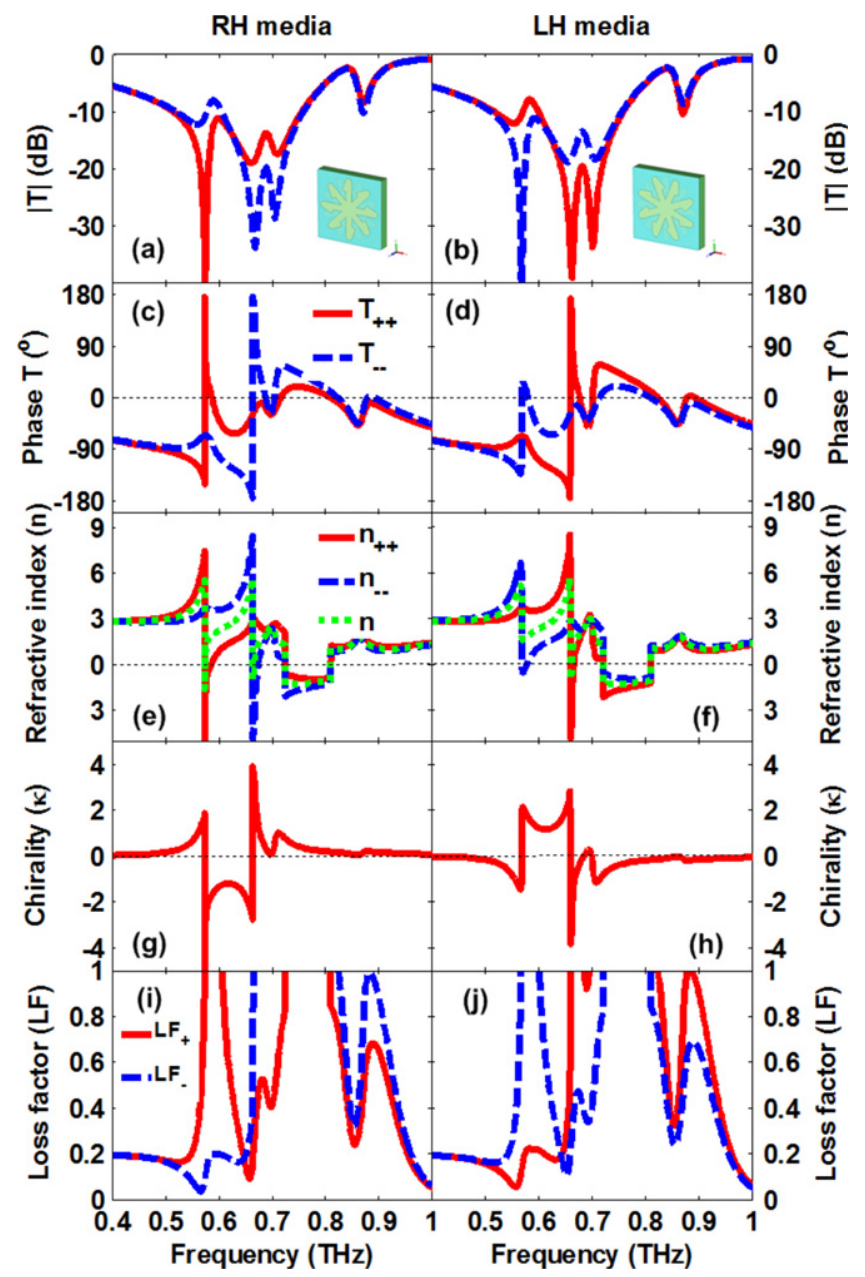

Figure 9. Properties of the conjugated bi-layer RH and LH C8 structures with lossless dielectrics in response to the circularly polarized waves. (Calculated) (a), (b) Transmission amplitude, (c), (d) transmission phase, (e), (f) refractive index, (g), (h) chirality, and (i), (j) loss factor.

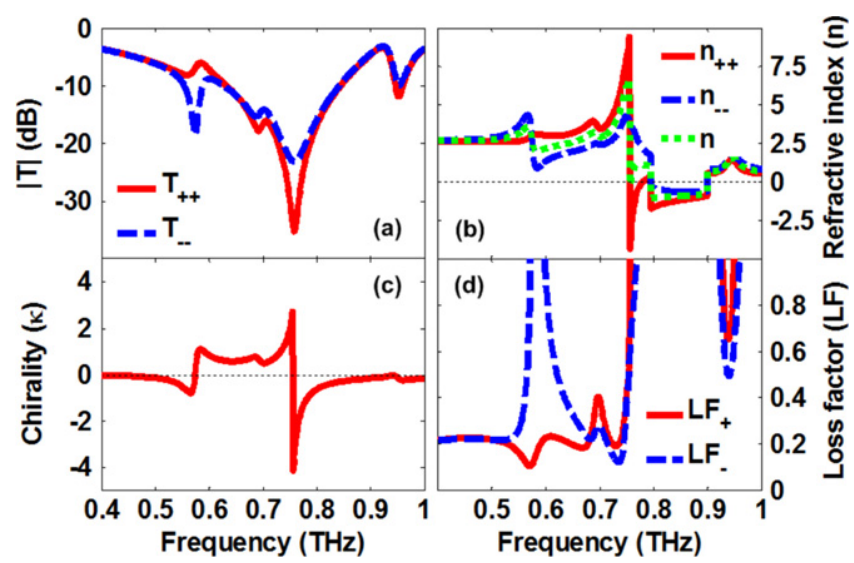

Figure 10. Properties of the conjugated bi-layer LH C8 structures with lossless dielectrics in response to the circularly polarized waves. (Calculated) (a) Transmission amplitude, (b) refractive index, (c) chirality, and (d) loss factor. 


\section{Conclusions}

The thin and flexible bi-layer CMMs, or the conjugated bilayered C8 structures, have been experimentally demonstrated at terahertz frequencies. Both the polarization changes and the antipallarel current in the asymmetric resonance mode at the observed frequency range support the chirality appearance of these proposed structures. These LH and RH structures are able to distinguish circularly polarized waves depending on the handedness of the structures, and the broad chirality band is observed. In order to enhance the polarization conversion efficiency, lower loss and thinner dielectrics used as a substrate and spacers are taken into consideration.

\section{Acknowledgments}

This project is supported by the Royal Golden Jubilee $\mathrm{PhD}$ program under the Thailand Research Fund (TRF), Grant No. PHD/0081/2554, the Faculty of Engineering, Khon Kaen University, Thailand, and the Thailand Research Fund (RSA5480010). PG acknowledges an Australian Government Endeavour International Postgraduate Research Scholarship. MB and SS acknowledge the Australian Research Council for funding via DP1092717 and DP110100262.

\section{References}

[1] Lindell I V, Sihvola A H, Tretyakov S A and Viitanen A J 1994 Electromagnetic Waves in Chiral and Bi-Isotropic Media (Boston: Artech House Publishers)

[2] Wongkasem N and Akyurtlu A 2010 Light splitting effects in chiral metamaterials J. Opt. 12035101

[3] Gansel J K, Thiel M, Rill M S, Decker M, Bade K, Saile V, Freymann G V, Linden S and Wegener M 2009 Gold helix photonic metamaterial as broadband circular polarizer Science 3251513

[4] Zhang S et al 2012 Photoinduced handedness switching in terahertz chiral metamolecules Nat. Commun. 3942

[5] Yang Y, da Costa R C, Fuchter M J and Campbell A J 2013 Circularly polarized light detection by a chiral organic semiconductor transistor Nat. Photonics 7634

[6] Demetriadou A and Pendry J B 2009 Extreme chirality in Swiss roll metamaterials J. Phys.: Condens. Matter. 21 376003

[7] Wiltshire M C K, Pendry J B and Hajnal J V 2009 Chiral Swiss rolls show a negative refractive index $J$. Phys.: Condens. Matter 21292201

[8] Sonsilphong A and Wongkasem N 2012 Three-dimensional artificial double helices with high negative refractive index J. Opt. 14105103

[9] Sonsilphong A and Wongkasem N 2013 Low loss circular birefringence in artificial triple helix Prog. Electromagn. Res. M 29 267-78

[10] Wang B, Zhou J, Koschny T and Soukoulis C M 2009 Nonplanar chiral metamaterials with negative index Appl. Phys. Lett. 94151112

[11] Plum E, Zhou J, Dong J, Fedotov V A, Th K, Soukoulis C M and Zheludev N I 2009 Metamaterial with negative index due to chirality Phys. Rev. B 79035407
[12] Matra K and Wongkasem N 2009 Left-handed chiral isotropic metamaterials: analysis and detailed numerical study J. Opt. A: Pure Appl. Opt. 11074011

[13] Panpradit W, Sonsilphong A, Soemphol C and Wongkasem N 2012 High negative refractive index in chiral metamaterials J. Opt. 14075101

[14] Wu J, Ng B, Turaga S P, Breese M B H, Maier S A, Hong M, Bettiol A A and Moser H O 2013 Free-standing terahertz chiral meta-foils exhibiting strong optical activity and negative refractive index Appl. Phys. Lett. 103141106

[15] Zhu W, Rukhlenko I D, Huang Y, Wen G and Premaratne M 2013 Wideband giant optical activity and negligible circular dichroism of near-infrared chiral metamaterial based on a complementary twisted configuration J. Opt. 15125101

[16] Cui Y, Kang L, Lan S, Rodrigues S and Cai W 2014 Chiral optical response from a twisted-arc metamaterial Nano Lett. 14 1021-5

[17] Choi M, Lee S H, Kim Y, Kang S B, Shin J, Kwak M H, Kang K-Y, Lee Y-H, Park N and Min B 2011 A terahertz metamaterial with unnaturally high refractive index Nature 470 369-74

[18] Zhou J, Chowdhury D R, Zhao R, Azad A K, Chen H-T, Soukoulis C M, Taylor A J and O'Hara J F 2012 Terahertz chiral metamaterials with giant and dynamically tunable optical activity Phys. Rev. B $\mathbf{8 6} 035448$

[19] Zhang S, Park Y-S, Li J, Lu X, Zhang W and Zhang X 2009 Negative refractive index in chiral metamaterials Phys. Rev. Lett. 102023901

[20] Lv T T, Zhu Z, Shi J H, Guan C Y, Wang Z P and Cui T J 2014 Optically controlled background-free terahertz switching in chiral metamaterial Opt. Lett. 39 3066-9

[21] Kenanakis G, Zhao R, Stavrinidis A, Konstantinidis G, Katsarakis N, Kafesaki M, Soukoulis C M and Economou E N 2012 Flexible chiral metamaterials in the terahertz regime: a comparative study of various designs Opt. Mater. Express 2 1702-12

[22] Gutruf P, Walia S, Nur Ali M, Sriram S and Bhaskaran M 2014 Strain response of stretchable micro-electrodes: controlling sensitivity with serpentine designs and encapsulation Appl. Phys. Lett. 104021908

[23] Li J, Shah C M, Withayachumnankul W, Ung B S-Y, Mitchell A, Sriram S, Bhaskaran M, Chang S and Abbott D 2013 Mechanically tunable terahertz metamaterials Appl. Phys. Lett. 102121101

[24] Piesiewicz R, Jansen C, Wietzke S, Mittleman D, Koch M and Kürner T 2007 Properties of building and plastic materials in the THz range Int. J. Infrared Millim. Waves 28 363-71

[25] Tao H, Strikwerda A C, Fan K, Bingham C M, Padilla W J, Zhang X and Averitt R D 2008 Terahertz metamaterials on free-standing highly-flexible polyimide substrates $J$. Phys. D: Appl. Phys. 41232004

[26] Wang B, Zhou J, Koschny T, Kafesaki M and Soukoulis C M 2009 Chiral metamaterials: simulations and experiments J. Opt. A: Pure Appl. Opt. 11114003

[27] Savenkov S N, Marienko V V, Oberemok E A and Sydoruk O 2006 Generalized matrix equivalence theorem for polarization theory Phys. Rev. E 74056607

[28] Zhou J, Dong J, Wang B, Koschny T, Kafesaki M and Soukoulis C M 2009 Negative refractive index due to chirality Phys. Rev. B 79 121104(R)

[29] Li Z, Zhao R, Koschny T, Kafesaki M, Alici K B, Colak E, Caglayan H, Ozbay E and Soukoulis C M 2010 Chiral metamaterials with negative refractive index based on four 'U' split ring resonators Appl. Phys. Lett. 97081901

[30] Zhao R, Zhang L, Zhou J, Koschny T and Soukoulis C M 2011 Conjugated gammadion chiral metamaterial with uniaxial optical activity and negative refractive index Phys. Rev. B 83 035105 